\title{
A Critical Phenomenon in Solitonic Ising Chains ${ }^{\star}$
}

\author{
Igor M. LOUTSENKO ${ }^{\dagger}$ and Vyacheslav P. SPIRIDONOV ${ }^{\ddagger}$ \\ $\dagger$ Mathematical Institute, University of Oxford, 24-29 St. Gilles', Oxford, OX1 3LB, UK \\ E-mail: loutsenk@maths.ox.ac.uk \\ $\ddagger$ Bogoliubov Laboratory of Theoretical Physics, JINR, Dubna, Moscow Region, 141980 Russia \\ E-mail: spiridon@theor.jinr.ru
}

Received December 04, 2006, in final form April 17, 2007; Published online April 24, 2007

Original article is available at http://www.emis.de/journals/SIGMA/2007/059/

\begin{abstract}
We discuss a phase transition of the second order taking place in non-local 1D Ising chains generated by specific infinite soliton solutions of the KdV and BKP equations.

Key words: Ising chain; solitons; phase transition
\end{abstract}

2000 Mathematics Subject Classification: 70H06; 82B20

To the memory of Vadim B. Kuznetsov

\section{The Korteweg-de Vries solitonic spin chain}

In a series of papers $[9,10]$, we described a direct relation between soliton solutions of integrable hierarchies and lattice gas systems (e.g., Coulomb gases on two dimensional lattices). The latter models can be reformulated also as some Ising spin systems with a non-local exchange. In particular, the grand partition functions of specific $N$-site Ising chains for some fixed values of the temperature were shown to coincide with the tau-functions of $N$-soliton solutions of the Korteweg-de Vries (KdV) and Kadomtsev-Petviashvili (KP) equations.

We would like to complete here the consideration of [9] and investigate a critical phenomenon appearing in these models in the zero temperature limit. The $N$-soliton solution of the $\mathrm{KdV}$ equation $u_{t}+u_{x x x}-6 u u_{x}=0$ has the form $[1,11]$

$$
u(x, t)=-2 \partial_{x}^{2} \log \tau_{N}(x, t),
$$

where $\tau_{N}$ is the determinant of a $N \times N$ matrix $M$,

$$
\begin{aligned}
& \tau_{N}=\operatorname{det} M, \quad M_{i j}=\delta_{i j}+\frac{2 \sqrt{k_{i} k_{j}}}{k_{i}+k_{j}} e^{\left(\theta_{i}+\theta_{j}\right) / 2}, \\
& \theta_{i}=k_{i} x-k_{i}^{3} t+\theta_{i}^{(0)}, \quad i, j=1,2, \ldots, N .
\end{aligned}
$$

The parameters $k_{i}$ describe amplitudes of solitons, $\theta_{i}^{(0)} / k_{i}$ are the zero time phases of solitons, and $k_{i}^{2}$ are their velocities. The tau-function $\tau_{N}$ admits the following Hirota type representation [7]:

$$
\tau_{N}=\sum_{\mu_{i}=0,1} \exp \left(\sum_{1 \leq i<j \leq N} A_{i j} \mu_{i} \mu_{j}+\sum_{i=1}^{N} \theta_{i} \mu_{i}\right),
$$

^This paper is a contribution to the Vadim Kuznetsov Memorial Issue 'Integrable Systems and Related Topics'. The full collection is available at http://www.emis.de/journals/SIGMA/kuznetsov.html 
where the soliton phase shifts $A_{i j}$ are expressed in terms of the spectral variables $k_{i}$ as

$$
e^{A_{i j}}=\frac{\left(k_{i}-k_{j}\right)^{2}}{\left(k_{i}+k_{j}\right)^{2}}
$$

As remarked in [9] for $\theta_{i}=\theta^{(0)}$, this $\tau_{N}$ defines the grand partition function of a lattice gas model with the chemical potential $\theta^{(0)}$ and $\mu_{i}$ being the filling factors of the lattice sites by molecules. The constants $A_{i j}$ describe interaction energy of the molecules.

Substituting in $(1) \mu_{i}=\left(\sigma_{i}+1\right) / 2$, where $\sigma_{i}= \pm 1$ are other discrete variables, one can pass from the lattice gases to Ising spin chains [3]:

$$
\tau_{N}=e^{\Phi} Z_{N}, \quad \Phi=\frac{1}{4} \sum_{i<j} A_{i j}+\frac{1}{2} \sum_{j=1}^{N} \theta_{j},
$$

where

$$
\begin{aligned}
& Z_{N}=\sum_{\sigma_{i}= \pm 1} e^{-\beta E}, \quad \beta=\frac{1}{k T}, \\
& E=\sum_{1 \leq i<j \leq N} J_{i j} \sigma_{i} \sigma_{j}-\sum_{i=1}^{N} H_{i} \sigma_{i} .
\end{aligned}
$$

Here, $J_{i j}$ are the exchange constants, $H_{i}$ is an external magnetic field, $T$ is the temperature, and $k$ is the Boltzmann constant:

$$
\beta J_{i j}=-\frac{1}{4} A_{i j}, \quad \beta H_{i}=\frac{1}{2} \theta_{i}+\frac{1}{4} \sum_{j=1, i \neq j}^{N} A_{i j} .
$$

$\tau_{N}$ coincides thus with the partition function of a one-dimensional Ising chain with the specific non-local exchange. A similar situation holds for the KP hierarchy and some other partial differential or difference nonlinear integrable equations.

From the thermodynamic point of view, it is interesting to understand the $N \rightarrow \infty$ behaviour of these "solitonic" statistical mechanics models. In general there are infinitely many free parameters, and it is difficult to classify qualitatively different cases. An interesting class of models is related to the so-called self-similar potentials [12]. These potentials are characterized by the $q$-periodicity constraints $k_{j+M}=q k_{j}$ and $\theta_{j+M}^{(0)}=\theta_{j}^{(0)}$, where $q$ is an arbitrary parameter, $0<q<1$, and $M$ is a positive integer. Exchange constants satisfy in this case the constraints $J_{i+M, j+M}=J_{i j}$. For $M=1$, this translational invariance takes the simplest form $J_{i j}=J(i-j)$ with $k_{i}$ forming one geometric progression, $k_{i}=k_{1} q^{i-1}, q=e^{-2 \alpha}$, where $k_{1}$ and $\alpha>0$ are free parameters. More precisely,

$$
A_{i j}=2 \log |\tanh \alpha(i-j)| .
$$

The KdV coordinate $x$ and time $t$ describe a part of the magnetic field $H_{i}$ decaying exponentially fast for $i \rightarrow \infty$ because $q<1$. Only the values of constants $\theta_{i}^{(0)}$ are therefore relevant for the leading asymptotics of the partition function in the $N \rightarrow \infty$ thermodynamic limit. Neglecting this $(x, t)$-dependence, we come to the constraint $H_{i+M}=H_{i}$, which is just the homogeneity condition for $M=1$. In principle, it is possible to compute the $N \rightarrow \infty$ asymptotics for the partition function for arbitrary $M$-periodic magnetic fields, but in [9] only the $M=1,2$ cases were considered.

Since $0<|\tanh \alpha(i-j)|<1$, we have $J_{i j}=-A_{i j} / 4 \beta>0$, which corresponds to an antiferromagnetic Ising chain (a similar picture holds for $M>1$ ). Although we have a long distance 
interaction, its intensity falls off exponentially fast, and the absence of phase transitions in such systems at non-zero temperatures is well known [5]. It appears, however, that there exists a special limit leading to a nontrivial critical phenomenon in this model. Indeed, we consider the limit $\alpha \rightarrow 0^{+}$or $q \rightarrow 1^{-}$. The phase shifts $A_{i j} \propto J_{i j} / k T$ then diverge. We can take nevertheless as the true exchange constants $J_{i j}^{\text {ren }}=J_{i j}\left(q^{-1}-q\right)$ and as the true temperature $k T_{\text {ren }}=k T\left(q^{-1}-q\right)$. For the self-consistency of the temperature definition, we should renormalize the magnetic field as well, $H \propto h /\left(q^{-1}-q\right)$, and assume that $h$ is finite.

As a result, the interaction energy of any spin "in the bulk" with all others,

$$
E_{i}=\sum_{j=-\infty, \neq i}^{\infty} J_{i j}^{\mathrm{ren}} \sigma_{j}
$$

is finite for $q \rightarrow 1^{-}$(or $\alpha \rightarrow 0^{+}$). Indeed, the maximal value of this interaction energy is

$$
E_{\max }=\lim _{\alpha \rightarrow 0} 4 \alpha \sum_{j=-\infty, \neq 0}^{\infty} J_{0 j}=-\frac{2}{\beta} \lim _{\alpha \rightarrow 0} \alpha \sum_{j=-\infty, \neq 0}^{\infty} \log \left|\frac{1-q^{j}}{1+q^{j}}\right|=-\frac{4}{\beta} \lim _{\alpha \rightarrow 0} \alpha \log \frac{(q ; q)_{\infty}}{(-q ; q)_{\infty}} .
$$

We use the notation $(a ; q)_{\infty}=\prod_{k=0}^{\infty}\left(1-a q^{k}\right)$ and $\left(a_{1}, \ldots, a_{m} ; q\right)_{\infty}=\prod_{j=1}^{m}\left(a_{j} ; q\right)_{\infty}$.

For the following considerations, we need theta functions [2]

$$
\begin{aligned}
& \theta_{1}(\nu, q)=-i \sum_{n \in \mathbb{Z}}(-1)^{n} q^{(n+1 / 2)^{2}} e^{(2 n+1) i \nu}=i q^{1 / 4} e^{-i \nu}\left(q^{2}, e^{2 i \nu}, q^{2} e^{-2 i \nu} ; q^{2}\right)_{\infty} \\
& \theta_{2}(\nu, q)=\sum_{n \in \mathbb{Z}} q^{(n+1 / 2)^{2}} e^{(2 n+1) i \nu}=q^{1 / 4} e^{-i \nu}\left(q^{2},-e^{2 i \nu},-q^{2} e^{-2 i \nu} ; q^{2}\right)_{\infty} \\
& \theta_{3}(\nu, q)=\sum_{n \in \mathbb{Z}} q^{n^{2}} e^{2 n i \nu}=\left(q^{2},-q e^{2 i \nu},-q e^{-2 i \nu} ; q^{2}\right)_{\infty} \\
& \theta_{4}(\nu, q)=\sum_{n \in \mathbb{Z}}(-1)^{n} q^{n^{2}} e^{2 n i \nu}=\left(q^{2}, q e^{2 i \nu}, q e^{-2 i \nu} ; q^{2}\right)_{\infty}
\end{aligned}
$$

where $q=e^{\pi i \tau}, \operatorname{Im}(\tau)>0$, and their modular transformations

$$
\begin{array}{ll}
\theta_{1}(\nu / \tau, \tilde{q})=-i \sqrt{-i \tau} e^{i \nu^{2} / \pi \tau} \theta_{1}(\nu, q), & \theta_{2}(\nu / \tau, \tilde{q})=\sqrt{-i \tau} e^{i \nu^{2} / \pi \tau} \theta_{4}(\nu, q), \\
\theta_{3}(\nu / \tau, \tilde{q})=\sqrt{-i \tau} e^{i \nu^{2} / \pi \tau} \theta_{3}(\nu, q), & \theta_{4}(\nu / \tau, \tilde{q})=\sqrt{-i \tau} e^{i \nu^{2} / \pi \tau} \theta_{2}(\nu, q),
\end{array}
$$

where $\tilde{q}=e^{-\pi i / \tau}$ and $\sqrt{-i \tau}$ is positive for purely imaginary $\tau$. Using these formulas, we obtain

$$
\frac{(q ; q)_{\infty}^{2}}{(-q ; q)_{\infty}^{2}}=\frac{\theta_{1}^{\prime}\left(0, q^{1 / 2}\right)}{\theta_{2}\left(0, q^{1 / 2}\right)}=\frac{\theta_{1}^{\prime}\left(0, \tilde{q}^{1 / 2}\right)}{(-i \tau) \theta_{4}\left(0, \tilde{q}^{1 / 2}\right)}=\tilde{q}^{1 / 8} \frac{2}{(-i \tau)} \frac{(\tilde{q} ; \tilde{q})_{\infty}^{2}}{\left(\tilde{q}^{1 / 2} ; \tilde{q}\right)_{\infty}^{2}}
$$

where $q=e^{2 \pi i \tau}=e^{-2 \alpha}$ and $\tilde{q}=e^{-2 \pi i / \tau}=e^{-2 \pi^{2} / \alpha}$. As a result,

$$
E_{\max }=\frac{\pi^{2}}{2 \beta}<\infty
$$

and $E_{i} \leq E_{\max }$. The limit $\alpha \rightarrow 0$ corresponds thus to an infinitely small and infinitely long-range nonlocal interaction model at a low value (zero) effective temperature.

There are some other interesting limits. For instance, for $q \rightarrow 0$ and finite $h$, we obtain the high temperature nearest neighbor interaction spin chain, $J_{i j}^{\text {ren }} \propto \delta_{i+1, j}, T_{\text {ren }} \rightarrow \infty$. For finite $H$, this limit corresponds to the non-interacting spins. The solitonic interpretation describes thus 
only a two-dimensional subspace of parameters $(T, H, q)$. For fixed $q$, the temperature $T$ is also fixed, and we can set the "KdV temperature" equal to $\beta=1$.

Using the Wronskian representation for $\tau_{N}$, the leading asymptotics of $Z_{N}$ for $N \rightarrow \infty$ was determined in [9] for the $M=1$ translationally invariant model and a homogeneous magnetic field. Namely, $Z_{N} \rightarrow \exp \left(-N \beta f_{I}\right)$, where the free energy per site $f_{I}$ has the form

$$
\begin{aligned}
& -\beta f_{I}(q, H)=\log \frac{2\left(q^{4} ; q^{4}\right)_{\infty} \cosh \beta H}{\left(q^{2} ; q^{2}\right)_{\infty}^{1 / 2}}+\frac{1}{4 \pi} \int_{0}^{2 \pi} d \nu \log \left(|\rho(\nu)|^{2}-q \tanh ^{2} \beta H\right), \\
& |\rho(\nu)|^{2}=\frac{\left(q^{2} e^{i \nu}, q^{2} e^{-i \nu} ; q^{4}\right)_{\infty}^{2}}{\left(q^{4} e^{i \nu}, q^{4} e^{-i \nu} ; q^{4}\right)_{\infty}^{2}} \frac{1}{4 \sin ^{2}(\nu / 2)}=q \frac{\theta_{4}^{2}\left(\nu / 2, q^{2}\right)}{\theta_{1}^{2}\left(\nu / 2, q^{2}\right)} .
\end{aligned}
$$

The total magnetization of the lattice takes the form:

$$
m(H)=-\frac{\partial f_{I}}{\partial H}=\lim _{N \rightarrow \infty} \frac{1}{N} \sum_{i=1}^{N}\left\langle\sigma_{i}\right\rangle=\left(1-\frac{1}{\pi} \int_{0}^{\pi} \frac{d \nu}{1+d(\nu) \cosh ^{2} \beta H}\right) \tanh \beta H,
$$

where

$$
d(\nu)=\frac{\theta_{4}^{2}\left(\nu, q^{2}\right)}{\theta_{1}^{2}\left(\nu, q^{2}\right)}-1 .
$$

The function $m(H)$ grows monotonically with $H$ and reflects qualitative predictions of the general theory of $1 \mathrm{D}$ systems with the fast decaying interactions [5]. However, the limit $\alpha \rightarrow 0$ with the renormalized exchange and magnetic field breaks down the corresponding necessary conditions, and we obtain a non-trivial critical phenomenon.

We substitute in (4) $\beta H=h /\left(q^{-1}-q\right), h>0$, and take the limit $\alpha \rightarrow 0$. Since

$$
\frac{\theta_{4}^{2}\left(\nu, q^{2}\right)}{\theta_{1}^{2}\left(\nu, q^{2}\right)}=-\frac{\theta_{2}^{2}\left(\nu / \tau, \tilde{q}^{2}\right)}{\theta_{1}^{2}\left(\nu / \tau, \tilde{q}^{2}\right)}=\frac{\left(-e^{2 i \nu / \tau},-\tilde{q}^{4} e^{-2 i \nu / \tau} ; \tilde{q}^{4}\right)_{\infty}^{2}}{\left(e^{2 i \nu / \tau}, \tilde{q}^{4} e^{-2 i \nu / \tau} ; \tilde{q}^{4}\right)_{\infty}^{2}}
$$

where $q=e^{\pi i \tau / 2}=e^{-2 \alpha}, \tilde{q}=e^{-\pi i / 2 \tau}=q^{-\pi^{2} / 8 \alpha}$, we have

$$
\begin{aligned}
\chi(\nu) & \equiv \lim _{\alpha \rightarrow 0} d(\nu) \cosh ^{2} \frac{h}{4 \alpha}=\lim _{\alpha \rightarrow 0}\left(\frac{\left(1+e^{2 i \nu / \tau}\right)^{2}\left(1+e^{2 i(\nu-\pi) / \tau}\right)^{2}}{\left(1-e^{2 i \nu / \tau}\right)^{2}\left(1-e^{2 i(\nu-\pi) / \tau}\right)^{2}}-1\right) \frac{e^{h / 2 \alpha}}{4} \\
& =\lim _{\alpha \rightarrow 0}\left(e^{-2 i \nu / \tau}+e^{2 i(\nu-\pi) / \tau}\right) e^{h / 2 \alpha}, \quad 0<\nu<\pi .
\end{aligned}
$$

Substituting this result in (4) and using relation $\lim _{\alpha \rightarrow 0} \tanh (h / 4 \alpha)=1$, we obtain

$$
m(h)=1-\frac{2}{\pi} \int_{0}^{\pi / 2} \frac{d \nu}{1+\chi(\nu)} .
$$

For $0<\nu<\pi / 2$, we have

$$
\chi(\nu)= \begin{cases}0, & \text { if } h / \pi<\nu<\pi / 2, \\ \infty, & \text { if } 0<\nu<h / \pi\end{cases}
$$

for $h<\pi^{2} / 2$, and $\chi(\nu)=\infty$, for $h>\pi^{2} / 2$. The final result therefore can be represented in the form

$$
m(h)=\left\{\begin{array}{lll}
1-\frac{2}{\pi} \int_{h / \pi}^{\pi / 2} d \nu=\frac{2}{\pi^{2}} h, & \text { if } & |h|<\pi^{2} / 2 \\
1, & \text { if } & |h| \geq \pi^{2} / 2
\end{array}\right.
$$


We have an obvious point of non-analyticity of $m(h)$ or of the free energy $f_{I}(h)$ at the critical value of the magnetic field $h_{\text {crit }}=\pi^{2} / 2$, such that the magnetic susceptibility $\chi(h)=$ $\beta^{-1} d m(h) / d h$ has a jump at it (i.e., we have the phase transition of the second order). This is a typical phenomenon in the systems with long-range interaction, where the mean field approximation gives exact values for the one-point correlation functions (see, e.g., [3]).

\section{The mean field approximation}

In the mean field theory, one considers a few degrees of freedom (usually, just one) of a taken system in an effective mean field of the remaining part of the system. This effective or mean field depends itself on the analysis of the one-body dynamics. As an example, we consider the general spin chain with energy (3) and the mean magnetization at the $i$-th site of the lattice

$$
\left\langle\sigma_{i}\right\rangle=\frac{\sum_{\sigma_{1}, \sigma_{2}, \ldots} \sigma_{i} e^{-\beta E}}{\sum_{\sigma_{1}, \sigma_{2}, \ldots} e^{-\beta E}}
$$

Instead of calculating the above sums, we stick to the $i$-th spin and evaluate its contribution to the energy as

$$
E_{i}\left(\sigma_{i}\right)=-\sigma_{i} \tilde{H}_{i}
$$

where

$$
\tilde{H}_{i}=-\sum_{j \neq i} J_{i j}\left\langle\sigma_{j}\right\rangle+H_{i}
$$

is an effective mean magnetic field at the $i$-th site created by the external field $H_{i}$ and the rest of the system $\propto \sum_{j \neq i} J_{i j}\left\langle\sigma_{j}\right\rangle$.

In the one body problem (5), the configuration space consists of two states $\sigma_{i}= \pm 1$, and therefore

$$
\left\langle\sigma_{i}\right\rangle=\frac{e^{\beta \tilde{H}_{i}}-e^{-\beta \tilde{H}_{i}}}{e^{\beta \tilde{H}_{i}}+e^{-\beta \tilde{H}_{i}}}=\tanh \left(\beta \tilde{H}_{i}\right) .
$$

Substituting the values of effective fields (6) in the last equation, we obtain a system of transcendental equations for mean values of all spins

$$
\left\langle\sigma_{i}\right\rangle=\tanh \beta\left(-\sum_{j \neq i} J_{i j}\left\langle\sigma_{j}\right\rangle+H_{i}\right)
$$

We consider now the translationally invariant system $J_{i j}=J(i-j), H_{i}=H$. In such a system, all mean values of the spins are the same in the thermodynamic limit, $\left\langle\sigma_{i}\right\rangle=\langle\sigma\rangle$, and from (7), we obtain

$$
\langle\sigma\rangle=\tanh \beta\left(-\sum_{j \neq i} J(i-j)\langle\sigma\rangle+H\right)=\tanh \beta(-J\langle\sigma\rangle+H), \quad J=\sum_{j \neq 0} J(j) .
$$

The solution of this equation is an intersection of graphs of two functions: $y=x$ and $y=$ $\tanh \beta(-J x+H)$. We consider two different cases. 
1) A ferromagnet in the zero magnetic field: $J<0, H=0$. Our system of equations has only the trivial solution $x=y=0$ for $\beta|J| \leq 1$ and three solutions $x=y=0$ and $x=y= \pm m$ for $\beta|J|>1$ and some $0<m<1$. The latter nontrivial solutions describe the spontaneous magnetization $m$ at the temperatures smaller than the critical value $1 / \beta_{\text {crit }}=|J|$ (the $\tanh (-\beta J x)$-function becomes steeper at the origin as $\beta$ increases and starts to intersect the line $x=y$ in two additional points as its slope exceeds the critical value).

2) An antiferromagnet, $J>0$. The only solution at $H=0$ is the trivial solution $x=y=0$. We consider now the zero-temperature limit $\beta \rightarrow \infty$. In this case, the tanh-function transforms to the sign-function, and we obtain

$$
\left\{\begin{array}{ll}
y=x, \\
y=\operatorname{sgn}(-J x+H),
\end{array} \operatorname{sgn}(x)=\left\{\begin{aligned}
1, & x>0 \\
-1, & x<0 .
\end{aligned}\right.\right.
$$

Solving these equations is rather easy. The function $\operatorname{sgn}(x)$ is shifted by $H / J$ from the origin along the $x$-axis. When $|H / J|<1$, it intersects with the line $y=x$ by its vertical part, and the magnetization equals to $H / J$. When $|H|$ exceeds $J$, the line $y=x$ intersects with one of the horizontal branches of $\operatorname{sgn}(x)$, and the magnetization becomes equal to \pm 1 . The magnetization is thus a continuous piecewise linear function of $H$ consisting of three parts: two constant $\langle\sigma\rangle= \pm 1$ for $|H|>J$ and the linear piece $\langle\sigma\rangle=H / J$ connecting them through the origin. In our $\mathrm{KdV}$-solitonic model, we denoted $\beta H=h /\left(q^{-1}-q\right)$ and $E_{\max }=\left(q^{-1}-q\right) J$. In the limit $q \rightarrow 1$, we have $E_{\max }=\pi^{2} / 2 \beta$ and $\langle\sigma\rangle=H / J=2 h / \pi^{2}$, which leads to the exact value of the critical magnetic field $h_{\text {crit }}=\pi^{2} / 2$. Such a qualitative behaviour of the system is obvious: when the external field exceeds the interaction energy between spins, they all flip in the field direction.

The mean field approximation is known to give exact one-point correlation functions (e.g., the magnetization) for systems with the long-range interaction (as our $q \rightarrow 1$ limit). It might be non-suitable, however, for the two point correlators (e.g., $\left.\left\langle\sigma_{i} \sigma_{j}\right\rangle\right)$.

We consider now the $M$-periodic chain. For general (not necessarily solitonic) $M$-periodic chain, it is reasonable to introduce multi-index exchange $J^{n m}(i-j)$, where $i-j$ is the distance between the cells and $1 \leq n, m \leq M$ are the respective internal cell indices for the $n$-th and $m$-th sublattices. In our particular solitonic KdV case, we have

$$
\beta J^{n m}(i)=-\frac{1}{2} \log \left|\frac{k_{n} q^{i}-k_{m}}{k_{n} q^{i}+k_{m}}\right|, \quad J^{n m}(i)=J^{m n}(-i)
$$

and the energy

$$
E=\sum_{i, j \in Z, n \neq m} J^{n m}(i-j) \sigma_{i}^{(n)} \sigma_{j}^{(m)}+\sum_{i, j \in Z, i \neq j, n} J^{n n}(i-j) \sigma_{i}^{(n)} \sigma_{j}^{(n)}+\sum_{i \in Z, n} H^{(n)} \sigma_{i}^{(n)} .
$$

In the latter sum the magnetic field is also $M$-periodic, $H_{i}^{(n)}=H^{(n)}$, inhomogeneous only inside the cells. The analysis similar to the $M=1$ case yields from (7) the following system of $M$ equations for $M$ unknowns $\left\langle\sigma^{(n)}\right\rangle$ :

$$
\left\langle\sigma^{(n)}\right\rangle=\tanh \beta\left(-\sum_{m} J^{n m}\left\langle\sigma^{(m)}\right\rangle+H^{(n)}\right), \quad n, m=1, \ldots, M .
$$

In these equations,

$$
J^{n n}=\sum_{i \in Z, \neq 0} J^{n n}(i), \quad J^{n m}=\sum_{i \in Z} J^{n m}(i), \quad n \neq m .
$$


In the zero-temperature limit, we have

$$
\left\langle\sigma^{(n)}\right\rangle=\operatorname{sgn}\left(-\sum_{m} J^{n m}\left\langle\sigma^{(m)}\right\rangle+H^{(n)}\right), \quad n, m=1, \ldots, M,
$$

where $\operatorname{sgn}(x)$ is the sign-function.

In the solitonic case (8), the $M \times M$ matrix $J=J^{n m}$ is symmetric with the constant diagonal:

$$
J^{n n}=-\frac{1}{2 \beta} \sum_{i \in Z, i \neq 0} \log \left|\frac{1-q^{i}}{1+q^{i}}\right| \equiv-A, \quad J^{n m}=J^{m n}=-\frac{1}{2 \beta} \sum_{i \in Z} \log \left|\frac{k_{n} q^{i}-k_{m}}{k_{n} q^{i}+k_{m}}\right| .
$$

For $M=2$, we have $J^{12}=J^{21} \equiv-B$ and

$$
\begin{aligned}
& \left\langle\sigma^{(1)}\right\rangle=\operatorname{sgn}\left(A\left\langle\sigma^{(1)}\right\rangle+B\left\langle\sigma^{(2)}\right\rangle+H^{(1)}\right), \\
& \left\langle\sigma^{(2)}\right\rangle=\operatorname{sgn}\left(B\left\langle\sigma^{(1)}\right\rangle+A\left\langle\sigma^{(2)}\right\rangle+H^{(2)}\right) .
\end{aligned}
$$

If we take the uniform magnetic field $H^{(1)}=H^{(2)}=H$, these equations become symmetric in $\sigma^{(1)}$ and $\sigma^{(2)}$ and have the solution $\langle\sigma\rangle=\left\langle\sigma^{(1)}\right\rangle=\left\langle\sigma^{(2)}\right\rangle$ stemming from one equation

$$
\langle\sigma\rangle=\operatorname{sgn}((A+B)\langle\sigma\rangle+H) .
$$

For the completely uniform magnetic field, there exists thus a solution when the spins flip simultaneously for both sublattices for sufficiently large magnetic fields.

Simultaneous phase transition exists for all sublattices in the uniform field $H^{(1)}=\cdots=$ $H^{(M)}=H$, when $\left\langle\sigma^{(1)}\right\rangle=\cdots=\left\langle\sigma^{(M)}\right\rangle$ and all spins in all sublattices are aligned simultaneously for a sufficiently large $H$. As seen from (9), such a solution exists, if $\sum_{m} J^{n m}$ are equal, which is certainly true for $M=2$ because of the permutational symmetry. But it may be not so for $M>2$. For instance, in the solitonic case (10) for $M=3$, we have

$$
\begin{aligned}
& \sum_{m} J^{1 m} \propto \sum_{i \in Z, \neq 0} \log \left|\frac{1-q^{i}}{1+q^{i}}\right|+\sum_{i \in Z} \log \left|\frac{k_{1} q^{i}-k_{2}}{k_{1} q^{i}+k_{2}}\right|+\sum_{i \in Z} \log \left|\frac{k_{1} q^{i}-k_{3}}{k_{1} q^{i}+k_{3}}\right|, \\
& \sum_{m} J^{2 m} \propto \sum_{i \in Z, \neq 0} \log \left|\frac{1-q^{i}}{1+q^{i}}\right|+\sum_{i \in Z} \log \left|\frac{k_{2} q^{i}-k_{1}}{k_{2} q^{i}+k_{1}}\right|+\sum_{i \in Z} \log \left|\frac{k_{2} q^{i}-k_{3}}{k_{2} q^{i}+k_{3}}\right|, \\
& \sum_{m} J^{3 m} \propto \sum_{i \in Z, \neq 0} \log \left|\frac{1-q^{i}}{1+q^{i}}\right|+\sum_{i \in Z} \log \left|\frac{k_{3} q^{i}-k_{1}}{k_{3} q^{i}+k_{1}}\right|+\sum_{i \in Z} \log \left|\frac{k_{3} q^{i}-k_{2}}{k_{3} q^{i}+k_{2}}\right| .
\end{aligned}
$$

These three sums are certainly different for $0<q<1$, and in the limit $\beta \rightarrow \infty$ (which we cannot reach within the solitonic interpretation for $q<1$ ), the magnetization would become a piecewise linear function of $H$ of a more complicated form than in the $M=1,2$ cases. In our model, however, the zero temperature is reached by multiplication of the above sums by $q^{-1}-q$ and taking the limit $q \rightarrow 1^{-}$(or $\alpha \rightarrow 0^{+}$). All three sums become then equal yielding the same magnetization as in the $M=1$ and $M=2$ cases.

\section{The BKP solitonic spin chain}

Another Ising chain model solved in [9] appears from the multisoliton solution of the KP equation of $B$ type, i.e. the BKP equation [4]. The corresponding partition function has the same form (2), where the exchange constants are

$$
\beta J_{i j}=-\frac{1}{4} A_{i j}, \quad e^{A_{i j}}=\frac{\left(a_{i}-a_{j}\right)\left(b_{i}-b_{j}\right)\left(a_{i}-b_{j}\right)\left(b_{i}-a_{j}\right)}{\left(a_{i}+a_{j}\right)\left(b_{i}+b_{j}\right)\left(a_{i}+b_{j}\right)\left(b_{i}+a_{j}\right)} .
$$


For $a_{i}=b_{i}=k_{i} / 2$, this model coincides with the $\mathrm{KdV}$-inspired model at the twice lower value of the temperature obtained after the change $\beta \rightarrow 2 \beta$.

The translational invariance of this spin chain, $J_{i j}=J(i-j)$, yields

$$
a_{i}=q^{i-1}, \quad b_{i}=b q^{i-1}, \quad q=e^{-2 \alpha},
$$

where we normalize $a_{1}=1$ and assume that $0<q<1$ as before. This gives the exchange

$$
\beta J_{i j}=-\frac{1}{4} \log \frac{\tanh ^{2} \alpha(i-j)-(b-1)^{2} /(b+1)^{2}}{\operatorname{coth}^{2} \alpha(i-j)-(b-1)^{2} /(b+1)^{2}}
$$

where the parameter $b$ is restricted to three regions (because of the $b \rightarrow 1 / b$ invariance): either $-1<b<-q$ (the ferromagnetic chain, $J_{i j}<0$ ), or $q<b \leq 1$ or $|b|=1, b \neq-1$ (the antiferromagnetic chain, $\left.J_{i j}>0\right)$.

In the thermodynamic limit $N \rightarrow \infty$, the free energy per site for the homogeneous magnetic field $H_{i}=H$ takes the form [9]:

$$
-\beta f_{I}(H)=\frac{1}{4} \log \frac{(q, q, b q, q / b ; q)_{\infty}}{(-q,-q,-b q,-q / b ; q)_{\infty}}+\frac{1}{4 \pi} \int_{0}^{2 \pi} d \nu \log |2 \rho(\nu)|
$$

where

$$
\rho(\nu)=\cosh 2 \beta H+\frac{(-q ; q)_{\infty}^{2}}{\left(-e^{i \nu},-q e^{-i \nu} ; q\right)_{\infty}}\left(\frac{\left(b^{-1} e^{i \nu}, q b e^{-i \nu} ; q\right)_{\infty}}{\left(b^{-1}, q b ; q\right)_{\infty}}+\frac{\left(b e^{i \nu}, q b^{-1} e^{-i \nu} ; q\right)_{\infty}}{\left(b, q b^{-1} ; q\right)_{\infty}}\right) .
$$

Taking the derivative with respect to $H$, we find the magnetization

$$
m(H)=\left(1-\frac{1}{\pi} \int_{0}^{\pi} \frac{d \nu}{1+d(\nu) \cosh 2 \beta H}\right) \tanh 2 \beta H
$$

where

$$
d(\nu)=2 \frac{\theta_{1}\left(\phi / 2, q^{1 / 2}\right)}{\theta_{2}\left(0, q^{1 / 2}\right)} \frac{\theta_{2}\left(\nu, q^{1 / 2}\right)}{\theta_{1}\left(\nu+\phi / 2, q^{1 / 2}\right)-\theta_{1}\left(\nu-\phi / 2, q^{1 / 2}\right)}
$$

with $b=e^{i \phi}$. For real $\phi$ we have $|b|=1$, the choice $\phi=i \gamma, 0<\gamma<2 \alpha$, yields $q<b<1$, and for $\phi=\pi+i \gamma$, we have $-1<b<-q$. The limit $b \rightarrow 1$ describes the magnetization for the "KdV-spin chain" at the twice lower value of the temperature. A simple test of this expression consists in the choice $b=-1$ corresponding to the non-interacting spins, $J_{i j}=0$. In this case $d(\nu)=1$, and we obtain $m(H)=\tanh \beta H$ as it should be for the free system.

We substitute now $q=e^{-2 \alpha}$ and $H=2 h /\left(q^{-1}-q\right)$ in (11) and consider the limit $\alpha \rightarrow 0^{+}$. The factor 2 in front of $h$ was chosen for coincidence of this model with the the KdV spin chain with the effective replacement $\beta \rightarrow 2 \beta$ (i.e., the twice lower value of the temperature). We apply the modular transformation to theta functions in (12) and obtain

$$
d(\nu)=2 \frac{\theta_{1}\left(\phi / 2 \tau, \tilde{q}^{1 / 2}\right)}{\theta_{4}\left(0, \tilde{q}^{1 / 2}\right)} \frac{\theta_{2}\left(\nu / \tau, \tilde{q}^{1 / 2}\right)}{e^{-i \frac{\nu \phi}{\pi \tau}} \theta_{1}\left(\frac{\nu+\phi / 2}{\tau}, \tilde{q}^{1 / 2}\right)-e^{i \frac{\nu \phi}{\pi \tau}} \theta_{1}\left(\frac{\nu-\phi / 2}{\tau}, \tilde{q}^{1 / 2}\right)},
$$

where $\tau=i \alpha / \pi$ and $\tilde{q}=e^{-2 \pi i / \tau}=e^{-2 \pi^{2} / \alpha}$. Denoting

$$
\chi(\nu)=\lim _{\alpha \rightarrow 0} d(\nu) \cosh \frac{4 q h}{1-q^{2}},
$$


we therefore obtain

$$
\chi(\nu)=\lim _{\alpha \rightarrow 0} \frac{\frac{1}{2} e^{-\frac{\pi \phi}{2 \alpha}}\left(1-e^{\frac{\pi \phi}{\alpha}}\right)\left(1-e^{\frac{\pi(2 \nu-\pi)}{\alpha}}\right) e^{\frac{h}{\alpha}}}{e^{-\frac{\pi \nu}{\alpha}\left(1+\frac{\phi}{\pi}\right)-\frac{\pi \phi}{2 \alpha}}\left(1-e^{2 \pi \frac{\nu+\phi / 2}{\alpha}}\right)-e^{-\frac{\pi \nu}{\alpha}\left(1-\frac{\phi}{\pi}\right)+\frac{\pi \phi}{2 \alpha}}\left(1-e^{2 \pi \frac{\nu-\phi / 2}{\alpha}}\right)} .
$$

In the region $\phi=i \gamma, 0<\gamma<2 \alpha$, we have

$$
\chi(\nu)=\lim _{\alpha \rightarrow 0} \frac{\sin (\pi \gamma / 2 \alpha)\left(1-e^{\frac{\pi}{\alpha}(2 \nu-\pi)}\right) e^{\frac{h}{\alpha}-\frac{\pi \nu}{\alpha}}}{2 \sin ((\pi / 2-\nu) \gamma / \alpha)} .
$$

Since $0<\gamma / \alpha<2$, the sin-factors do not influence the asymptotic behaviour, and for $0<\nu<$ $\pi / 2$, we find $\chi(\nu)=0$ for $h / \pi<\nu<\pi / 2$ and $\chi(\nu)=\infty$ for $\nu<h / \pi$. As a result, we obtain $m(h)=2 h / \pi^{2}$ for $|h|<\pi^{2} / 2$ and $m(h)=1$ for $|h| \geq \pi^{2} / 2$. This is the same picture as for the "KdV-chain", as it should be because the limit $\alpha \rightarrow 0$ assumes the limit $\gamma \rightarrow 0$ or $b \rightarrow 1$.

In a similar way, for $\phi=\pi+i \gamma, 0 \leq \gamma<2 \alpha$, and $\alpha \rightarrow 0$, we find $b \rightarrow-1$, i.e. the trivial situation of free spins. The most interesting behaviour appears in the region $0<\phi<\pi$, for which we find

$$
m(h)=1-\frac{2}{\pi} \int_{0}^{\pi / 2} \frac{d \nu}{1+\chi(\nu)}
$$

where

$$
\chi(\nu)= \begin{cases}0, & \text { if } \quad h /(\pi-\phi)<\nu \\ \infty, & \text { if } \quad \nu<h /(\pi-\phi) .\end{cases}
$$

As a result,

$$
m(h)=\frac{2 h}{\pi(\pi-\phi)}, \quad \text { if } \quad|h|<\frac{\pi(\pi-\phi)}{2},
$$

and $m(h)=1$, if $|h| \geq \pi(\pi-\phi) / 2$. The critical value of the magnetic field, for which we have the phase transition,

$$
h_{\mathrm{crit}}= \pm \frac{\pi(\pi-\phi)}{2}
$$

depends explicitly on the parameter of the model $\phi$, and for $\phi=0$, we obtain the previous result. The Ising spin systems associated with the multisoliton solutions of integrable nonlinear equations provide thus the models with phase transitions already in their simplest one-dimensional spin chain realizations.

It is interesting to analyze consequences of the antiferromagnetic nature of the exchange. We take for this the $\mathrm{KdV}$-inspired Ising chain and apply different magnetic fields to the odd, $H_{1}$, and even, $\mathrm{H}_{2}$, sites. The corresponding magnetization for the odd sites sublattice was derived in $[9]$ :

$$
\begin{aligned}
m_{\text {odd }}\left(H_{1}, H_{2}\right) & =-2 \frac{d f_{I}}{d H_{1}}=\lim _{p \rightarrow \infty} \frac{1}{p} \sum_{j=1}^{p}\left\langle\sigma_{2 j-1}\right\rangle \\
& =\tanh \beta H_{1}-\frac{\tanh \beta H_{2}}{\cosh ^{2} \beta H_{1}} \frac{1}{\pi} \int_{0}^{\pi} \frac{d \nu}{\frac{\theta_{4}^{2}\left(\nu, q^{2}\right)}{\theta_{1}^{2}\left(\nu, q^{2}\right)}-\tanh \beta H_{1} \tanh \beta H_{2}} .
\end{aligned}
$$


The magnetization for the even sites sublattice is obtained after permuting $H_{1}$ and $H_{2}$ in this expression. Obviously, if we take the alternating magnetic field $H_{1}=-H_{2}=H$, then the total magnetization is equal to zero, though the sublattice magnetizations remain non-trivial:

$$
m_{\text {odd }}(H)=\left(1+\frac{1}{\pi} \int_{0}^{\pi} \frac{d \nu}{\frac{\theta_{4}^{2}\left(\nu, q^{2}\right)}{\theta_{1}^{2}\left(\nu, q^{2}\right)} \cosh ^{2} \beta H+\sinh ^{2} \beta H}\right) \tanh \beta H .
$$

However, after substituting $\beta H=h /\left(q^{-1}-q\right)$ and taking the limit $\alpha \rightarrow 0$, we see that our zero temperature critical phenomenon disappears: $m_{\text {odd }}(h)=1$ for $h>0$ and $m_{\text {odd }}(h)=-1$ for $h<0$, similar to the free spins system.

\section{Conclusion}

As shown in [10], soliton solutions of integrable hierarchies with the complex values of spectral variables are connected to the intrinsic Coulomb gases on two dimensional lattices with some nontrivial dielectric or conductor boundaries. In this picture, the Coulomb interaction energy between two charges and their effective images created by the boundary conditions plays the role of the soliton phase shifts, the coordinates of charges coincide with the spectral parameters of solitons, and the external electrostatic field is expanded in some series with the coefficients playing the role of integrable hierarchy times. This transparent relation serves as a clue for building new Coulomb lattice gas models exactly solvable at some fixed temperatures. The latter temperatures are also related to the random matrix models $[6,9,10]$, but we do not discuss here applications of the described phase transition within these interpretations.

Our phase transitions are of a rather simple nature. In the lattice gas language, the transition in the $M=1$ periodic case describes the situation when the lattice is filled to its limit, i.e. the number of particles equals to the number of sites, and no more particles can be added to the system. In the $M$-periodic case, such transitions may happen separately, when each sublattice is filled completely one by one, or simultaneously, for an appropriate choice of parameters. Their qualitative features can be found from the mean field theory. There are several interesting questions which would be interesting to analyze in the future, like influences of the hierarchy times on the thermodynamical quantities, understanding of our systems beyond the "solitonic" temperature values, investigation of the higher correlation functions, and so on.

\section{Acknowledgements}

The work of I.M. Loutsenko has been supported by European Community grant MIFI-CT-2005007323 and V.P. Spiridonov is partially supported by the Russian Foundation for Basic Research (grant no. 05-01-01086). The authors are grateful to V.B. Priezzhev for useful remarks.

I vaguely remember a tall man with glasses vigorously explaining to me something during my poster presentation at the IGTMP colloquium in Moscow in 1990. Probably that was Vadim I never asked him about that later on. We got acquainted at the first SIDE meeting near Montréal in 1994 and had sufficiently long discussions during his few days visit to CRM after that conference. I remember telling him that by the work on separation of variables [8], which impressed me much, he closed to me that field, and it is necessary to think about other directions of research. We became closer during Vadim's stay at the CRM in 1994-1995. It was very nice time from many points of view. I visited his house and have known his family during one of the parties he was gathering. It appeared that he likes Russian "bards" singing, which I was bond to as well. Once he even sent to me a web-link to some new mp3-recordings coming from his native Saint-Petersburg. 
In June 1999, he chaired my talk at the Hong Kong meeting on special functions, where I reported results of the paper [13]. He was interested by that much, and we discussed possible intersections with integrable systems. I saw Vadim last time at the conference in Edinburgh in September 2003, where he was the main organizer. During the preparation of the corresponding proceedings, I actively communicated with him, and it was clear that he is extremely busy by all kinds of obligations. In May 2005, I suggested to him to form a team in order to try to get an INTAS grant. His first reaction was positive, but after he has known the rules and procedures, he rejected this idea by saying that there is too much bureaucracy and he has too many other commitments for the next few months. I totally agreed with his critics and accepted his excuse. Later on he listed to me a number of other possibilities to get research funding from the UK sources which sounded quite reasonable. We exchanged by about ten e-mails with him over the May-October 2005 period, and it was devastating to know that he has passed away. We have another deeply regrettable loss in the FSU scientific community, which was possible, probably, to prevent in other circumstances.

V.P. Spiridonov

I have met Vadim first when I have been pursuing my PhD studies at the Centre de Recherches Mathématiques in Montréal. At that time, Vadim was a postdoctoral fellow there. I remember him as an open-heart person, frank and honest, very enthusiastic and completely devoted to the problems he did and had in mind. Always full of energy, he showed the keenest interest for many questions of science and life. To meet Vadim was very interesting to me.

I.M. Loutsenko

\section{References}

[1] Ablowitz M.J., Segur H., Solitons and the inverse scattering transform, SIAM, Philadelphia, 1981.

[2] Andrews G.E., Askey R., Roy R., Special functions, Encyclopedia of Math. Appl., Vol. 71, Cambridge Univ. Press, Cambridge, 1999.

[3] Baxter R.J., Exactly solved models in statistical mechanics, Academic Press, London, 1982.

[4] Date E., Jimbo M., Kashiwara M., Miwa T., Transformation groups for soliton equations, in Nonlinear Integrable Systems, World Scientific, Singapore, 1983, 41-119.

[5] Evans M. R., Phase transitions in one-dimensional nonequilibrium systems, cond-mat/0007293.

[6] Gaudin M., Une famille à une paramètre d'ensembles unitaires, Nucl. Phys. 85 (1966), 545-575.

Gaudin M., Gaz coulombien discret à une dimension, J. Phys. (France) 34 (1973), 511-522.

[7] Hirota R., Exact solution of the Korteweg-de Vries equation for multiple collisions of solitons, Phys. Rev. Lett. 27 (1971), 1192-1194.

[8] Kuznetsov V.B., Quadrics on real Riemannian spaces of constant curvature: separation of variables and connection with Gaudin magnet, J. Math. Phys. 33 (1992), 3240-3254.

[9] Loutsenko I.M., Spiridonov V.P., Self-similar potentials and Ising models, Pis'ma v ZhETF (JETP Letters) 66 (1997), 747-753.

Loutsenko I.M., Spiridonov V.P., Spectral self-similarity, one-dimensional Ising chains and random matrices, Nucl. Phys. B 538 (1999), 731-758.

[10] Loutsenko I.M., Spiridonov V.P., Soliton solutions of integrable hierarchies and Coulomb plasmas, J. Stat. Phys. 99 (2000), 751-767, cond-mat/9909308.

[11] Matveev V.B., Salle M.A., Darboux transformations and solitons, Springer Series in Nonlinear Dynamics, Springer-Verlag, 1991.

[12] Spiridonov V.P., Universal superpositions of coherent states and self-similar potentials, Phys. Rev. A 52 (1995), 1909-1935, quant-ph/9601030.

[13] Spiridonov V.P., Zhedanov A.S., Spectral transformation chains and some new biorthogonal rational functions, Comm. Math. Phys. 210 (2000), 49-83. 